\title{
CENTRALIZERS OF EXPANDING MAPS ON THE CIRCLE
}

\author{
CARLOS ARTEAGA
}

(Communicated by Kenneth R. Meyer)

\begin{abstract}
We prove here that the elements of an open and dense subset of expanding maps on the circle have trivial centralizers; i.e., the maps commute only with their own powers. Using a theorem proved in [1], we deduce that the result is also true for an open and dense subset of immersions of $S^{1}$.
\end{abstract}

\section{INTRODUCTION}

Let $\operatorname{Imm}^{n}\left(S^{1}\right)$ be the space of $C^{n}$ immersions of the circle $S^{1}$ (i.e., mappings $f$ for which $f^{\prime}(x) \neq 0$ ) endowed with the $C^{n}$ topology. An immersion $f: S^{1} \rightarrow S^{1}$ is expanding iff $\left|f^{\prime}(x)\right|>1$ for all $x \in S^{1}$. Let $\operatorname{Exp}^{n}\left(S^{1}\right)$ denote the set of $C^{n}$ expanding maps of $S^{1}$.

We continue here to study, initiated in [1], of centralizers of immersions of the circle. Recall that for $f \in \operatorname{Imm}^{n}\left(S^{1}\right)$, its centralizer $Z(f)$ in $\operatorname{Imm}^{n}\left(S^{1}\right)$ is defined as the set of elements that commute with $f$. We say that $f$ has trivial centralizer if $Z(f)$ is reduced to the iterates $\left\{f^{n}, n \in \mathbf{N}\right\}$ of $f$.

The purpose of this paper is to prove the following result.

Theorem. For an open and dense subset of $\operatorname{Exp}^{n}\left(S^{1}\right)(n>1)$, the centralizer is trivial.

It follows from [1] that a similar result is true for an open and dense subset of $\operatorname{Imm}^{\infty}\left(S^{1}\right)-\overline{\operatorname{Exp}^{\infty}\left(S^{1}\right)}$. Hence we have proved

Corollary. There is an open and dense subset of $\operatorname{Imm}^{\infty}\left(S^{1}\right)$ whose elements have trivial centralizers.

This result is an extension to immersions of a theorem of Kopell [2, Theorem 3 ], who showed the triviality of the centralizer for an open and dense subset of diffeomorphisms of the circle.

A fundamental tool for the proof of the theorem is [1, Lemma 2.1] which is an extension to expanding maps on the circle of a result of Kopell [2, Theorem 6].

Received by the editors June 13, 1990.

1980 Mathematics Subject Classification (1985 Revision). Primary 58F15.

Key words and phrases. Expanding maps, centralizer, trivial centralizer.

The author was partially supported by CNPq, Brazil. 


\section{PRoOF OF THE THEOREM}

We begin by recalling some basic concepts and establishing preliminary results.

Formally, we will think of the circle as $\mathbf{R} / \mathbf{Z}$ and use $\pi$ to denote the canonical projection. Thus every continuous map $f$ of the circle has countably many lifts, i.e., continuous maps $F: S^{1} \rightarrow S^{1}$ satisfying

$$
f \circ \pi=\pi \circ F \text {. }
$$

Any two such lifts differ by an integer, and the unique integer $d$ satisfying

$$
F(x+1)=F(x)+d
$$

for all lifts $F$ and all $x$ is called the degree of $f$.

Let $f_{d}$ denote the immersion of $S^{1}$ given by $f_{d}(z)=z^{d}$.

Lemma 2.1. Let $f$ be an expanding map of the circle, and suppose that $f^{\prime}(x) \neq$ $f^{\prime}(y)$ for all different fuxed points of $f$. Then

(a) If $g \in Z(f)$, then $g$ fixes the fixed points of $f$.

(b) Let $h: S^{1} \rightarrow S^{1}$ be a homeomorphism of the circle such that $h \circ f=$ $f_{n} \circ h$, where $n=$ degree $f$. If $g \in Z(f)$, then $h \circ g=f_{m} \circ h$, where $m=$ degree $g$.

Proof. (a) Let $x$ be a fixed point of $f$. Then

$$
f \circ g(x)=g \circ f(x)=g(x),
$$

and

$$
f^{\prime}(g(x))=f^{\prime}(x) .
$$

These properties and the hypothesis imply that $g(x)=x$.

(b) We have that

$$
f_{n} \circ\left(h \circ g \circ h^{-1}\right)=h \circ f \circ g \circ h^{-1}=h \circ g \circ f \circ h^{-1}=\left(h \circ g \circ h^{-1}\right) \circ f_{n} \text {. }
$$

Hence, by [1, Lemma 2.1], $h \circ g \circ h^{-1}=\omega f_{m}$, where $\omega$ is an $(n-1)$ th root of unity and $m=$ degree $g$. By (a), $h^{-1}(1)$ is a fixed point of $g$. Thus

$$
1=h \circ g \circ h^{-1}(1)=\omega f_{m}(1)=\omega \text {. }
$$

Therefore, $h \circ g=f_{m} \circ h$, and the lemma is proved.

We will use the following simple fact.

Lemma 2.2. Let $f: S^{1} \rightarrow S^{1}$ be a $C^{n}$ endomorphism of the circle, and let $\alpha: S^{1} \rightarrow S^{1}$ be a $C^{n}$ diffeomorphism. If $Z\left(\alpha^{-1} \circ f \circ \alpha\right)$ is trivial, then $Z(f)$ is trivial.

Proof. Let $g \in Z(f)$. Then $\alpha^{-1} \circ g \circ \alpha \in Z\left(\alpha^{-1} \circ f \circ \alpha\right)$. Hence, by hypothesis, $\alpha^{-1} \circ g \circ \alpha=\alpha^{-1} \circ f^{k} \circ \alpha$ for some $k \in \mathbf{N}$. Thus, $g=f^{k}$ and so $Z(f)$ is trivial.

We are now ready to prove the theorem. Let

$$
\mathscr{U}=\left\{f \in \operatorname{Exp}^{n}\left(S^{1}\right): f^{\prime}(x) \neq f^{\prime}(y) \text { for different fixed points } x, y \text { of } f\right\} .
$$


This set is clearly an open and dense subset of $\operatorname{Exp}^{n}\left(S^{1}\right)$. Hence, it suffices to show that the elements of $\mathscr{U}$ have trivial centralizers. Let $f \in \mathscr{U}$. By a result of Shub [3], there exists an order-preserving homeomorphism $h: S^{1} \rightarrow S^{1}$ such that

$$
h \circ f=f_{n} \circ h, \quad \text { where } n=\operatorname{degree} f \text {. }
$$

By using a rotation of $S^{1}$, if necessary, we may assume by Lemma 2.2 that $f(1)=h(1)=1$. Let $g \in Z(f)$. By Lemma 2.1(a), $g(1)=1$. Hence, if $F, G, H$ are the lifts of $f, g$, and $h$, respectively, then

$$
\begin{aligned}
F(0) & =G(0)=H(0)=0, \\
H \circ F(x) & =n H(x) \quad \text { for all } x \in \mathbf{R},
\end{aligned}
$$

and by Lemma 2.1(b), $H \circ G(x)=m H(x)$ where $m=$ degree $g$. Let $l \in \mathbf{N}$ such that $n^{l} \leq m \leq n^{l+1}$. Then

$$
m=n^{l}+p \text { for some } 0 \leq p \leq n^{l}(n-1) .
$$

We claim that $G^{\prime}(0)=\left(F^{\prime}(0)\right)^{l}$. In fact, let $t_{k} \in \mathbf{R}$ such that $H\left(t_{k}\right)=1 / n^{k}$. Let $\varepsilon>0$ be given. Since $H$ is an order-preserving homeomorphism, we can choose $k \in \mathbf{N}$ such that

$$
0 \leq H^{-1}\left(\frac{1}{n^{k}}+\frac{p}{n^{k+l}}\right)-t_{k} \leq \varepsilon .
$$

Since $F^{-1}$ is a contraction, we have that

$$
\lim _{j \rightarrow \infty} F^{-j}\left(H^{-1}\left(\frac{1}{n^{k}}+\frac{p}{n^{k+l}}\right)\right)=0
$$

and

$$
0 \leq F^{-j}\left(H^{-1}\left(\frac{1}{n^{k}}+\frac{p}{n^{k+l}}\right)\right)-F^{-j}\left(t_{k}\right) \leq \varepsilon
$$

for all $j \in \mathbf{N}$. Thus

$$
1 \leq \frac{F^{-j}\left(H^{-1}\left(\frac{1}{n^{k}}+\frac{p}{n^{k+1}}\right)\right)}{F^{-j}\left(t_{k}\right)} \leq 1+\varepsilon \text { for all } j \in \mathbf{N} .
$$

Let $y_{i}=F^{-j}\left(t_{k}\right)$. Then

$$
\lim _{j \rightarrow \infty} y_{j}=0
$$

and

$$
\frac{1}{n^{k}}=H\left(t_{k}\right)=H \circ F^{j}\left(y_{j}\right)=n^{j} H\left(y_{j}\right) .
$$

Hence, $H\left(y_{j}\right)=1 / n^{k+j}$ for all $j \in \mathbf{N}$. These properties, together with the fact that $F^{-1} \circ H^{-1}(n x)=H^{-1}(x)$ for all $x \in \mathbf{R}$, imply that

$$
\begin{aligned}
G^{\prime}(0) & =\lim _{j \rightarrow \infty} \frac{G\left(y_{j}\right)-G(0)}{y_{j}}=\lim _{j \rightarrow \infty} \frac{H^{-1}\left(m H\left(y_{j}\right)\right)}{y_{j}} \\
& =\lim _{j \rightarrow \infty} \frac{H^{-1}\left(\frac{m}{n^{k+j}}\right)}{y_{j}}=\lim _{j \rightarrow \infty} \frac{F^{-(j-l)} \circ H^{-1}\left(\frac{m n^{j-l}}{n^{k+j}}\right)}{y_{j}} \\
& =\lim _{j \rightarrow \infty} \frac{F^{-(j-l)} \circ H^{-1}\left(\frac{n^{j}+p n^{j-l}}{n^{k+j}}\right)}{y_{j}} \\
& =\lim _{j \rightarrow \infty} \frac{F^{l}\left(F^{-j}\left(H^{-1}\left(\frac{1}{n^{k}}+\frac{p}{n^{k+1}}\right)\right)\right)}{F^{-j}\left(H^{-1}\left(\frac{1}{n^{k}}+\frac{p}{n^{k+1}}\right)\right)} \frac{F^{-j}\left(H^{-1}\left(\frac{1}{n^{k}}+\frac{p}{n^{k+1}}\right)\right)}{F^{-j}\left(t_{k}\right)} .
\end{aligned}
$$


But, by $(*)$,

$$
\lim _{j \rightarrow \infty} \frac{F^{l}\left(F^{-j}\left(H^{-1}\left(\frac{1}{n^{k}}+\frac{p}{n^{k+l}}\right)\right)\right)}{F^{-j}\left(H^{-1}\left(\frac{1}{n^{k}}+\frac{p}{n^{k+l}}\right)\right)}=\left(F^{l}\right)^{\prime}(0) .
$$

So, by $(* *)$,

$$
\left(F^{l}\right)^{\prime}(0) \leq G^{\prime}(0) \leq\left(F^{l}\right)^{\prime}(0)+\varepsilon .
$$

Therefore $G^{\prime}(0)=\left(F^{l}\right)^{\prime}(0)$, and the claim is proved.

By Sternberg [4], there exists a $C^{n-1}$-diffeomorphism $\alpha: \mathbf{R} \rightarrow \mathbf{R}$ such that $\alpha(0)=0$ and $\alpha \circ F \circ \alpha^{-1}(x)=F^{\prime}(0) x$ for all sufficiently small $x$. Let $L=$ $\alpha \circ F \circ \alpha^{-1}$. Then $\alpha \circ G \circ \alpha^{-1}$ commutes with $L$. Thus, for all sufficiently small $x$, we have that

$$
\begin{aligned}
\left(\alpha \circ G \circ \alpha^{-1}\right)(x) F^{\prime}(0) & =\left(\alpha \circ G \circ \alpha^{-1}\right)\left(L\left(\frac{x}{F^{\prime}(0)}\right)\right) L^{\prime}\left(\frac{x}{F^{\prime}(0)}\right) \\
& =L^{\prime}\left(\alpha \circ G \circ \alpha^{-1}\left(\frac{x}{F^{\prime}(0)}\right)\right)\left(\alpha \circ G \circ \alpha^{-1}\right)^{\prime}\left(\frac{x}{F^{\prime}(0)}\right) \\
& =F^{\prime}(0)\left(\alpha \circ G \circ \alpha^{-1}\right)^{\prime}\left(\frac{x}{F^{\prime}(0)}\right),
\end{aligned}
$$

so

$$
\left(\alpha \circ G \circ \alpha^{-1}\right)^{\prime}(x)=\left(\alpha \circ G \alpha^{-1}\right)^{\prime}\left(\frac{x}{F^{\prime}(0)}\right) .
$$

This and the fact that $\left|F^{\prime}(0)\right|>1$ imply that

$$
\left(\alpha \circ G \circ \alpha^{-1}\right)^{\prime}(x)=\left(\alpha \circ G \circ \alpha^{-1}\right)^{\prime}\left(\frac{x}{\left(F^{\prime}(0)\right)^{q}}\right) \quad \text { for all } q \in \mathbf{N} \text {. }
$$

Since $\left(\alpha \circ G \circ \alpha^{-1}\right)^{\prime}$ is continuous, we have that

$$
\left(\alpha \circ G \circ \alpha^{-1}\right)^{\prime}(x)=\left(\alpha \circ G \circ \alpha^{-1}\right)^{\prime}(0)=G^{\prime}(0) .
$$

This and the claim above imply that, for all sufficiently small $x$,

$$
\alpha \circ G \circ \alpha^{-1}(x)=G^{\prime}(0) x=\left(F^{\prime}(0)\right)^{l} x=L^{l}(x),
$$

so $G(x)=F^{l}(x)$ for all sufficiently small $x$. It follows from this that there exists an open interval $I$ in $S^{1}$ such that $g(x)=f^{l}(x)$ for all $x \in I$. Since $f$ is expanding, for all $y \in S^{1}$ there exist $x \in I$ and $q \in \mathbf{N}$ such that $f^{q}(x)=y$. Thus

$$
g(y)=g \circ f^{q}(x)=f^{q} \circ g(x)=f^{q} \circ f^{l}(x)=f^{l}(y) .
$$

Therefore $g=f^{l}$, and the theorem is proved.

\section{ACKNOWLEDGMENT}

The author expresses his gratitude to the referee for valuable suggestions about this paper.

\section{REFERENCES}

1. C. Arteaga, Centralizers of immersions of the circle, Proc. Amer. Math. Soc. 109 (1990), 849-853.

2. N. Kopell, Commuting diffeomorphisms, Proc. Sympos. Pure Math., vol. 14, Amer. Math. Soc., Providence, RI, 1970, pp. 165-184. 
3. M. Shub, Endomorphisms of compact differentiable manifolds, Amer. J. Math. 91 (1969), 175-199.

4. S. Sternberg, Local $C^{n}$ transformations of the real line, Duke Math. J. 24 (1957), 97-102.

Departamento de Matematica, Universidade Federal de São Carlos, 13560 São Carlos São Paulo, Brazil 